\title{
On Complete Convergence of Weighted Sums for Arrays of Rowwise Asymptotically Almost Negatively Associated Random Variables
}

\author{
Xuejun Wang, Shuhe Hu, Wenzhi Yang, and Xinghui Wang \\ School of Mathematical Science, Anhui University, Hefei 230039, China
}

Correspondence should be addressed to Xinghui Wang, wangxinghuial@163.com

Received 9 March 2012; Accepted 18 May 2012

Academic Editor: Irena Rachůnková

Copyright (C) 2012 Xuejun Wang et al. This is an open access article distributed under the Creative Commons Attribution License, which permits unrestricted use, distribution, and reproduction in any medium, provided the original work is properly cited.

Let $\left\{X_{n i}, i \geq 1, n \geq 1\right\}$ be an array of rowwise asymptotically almost negatively associated (AANA, in short) random variables. The complete convergence for weighted sums of arrays of rowwise AANA random variables is studied, which complements and improves the corresponding result of Baek et al. (2008). As applications, the Baum and Katz type result for arrays of rowwise AANA random variables and the Marcinkiewicz-Zygmund type strong law of large numbers for sequences of AANA random variables are obtained.

\section{Introduction}

The concept of complete convergence was introduced by Hsu and Robbins [1] as follows. A sequence of random variables $\left\{U_{n}, n \geq 1\right\}$ is said to converge completely to a constant $C$ if $\sum_{n=1}^{\infty} P\left(\left|U_{n}-C\right|>\varepsilon\right)<\infty$, for all $\varepsilon>0$. In view of the Borel-Cantelli lemma, this implies that $U_{n} \rightarrow C$ almost surely (a.s.). The converse is true if the $\left\{U_{n}, n \geq 1\right\}$ are independent. Hsu and Robbins [1] proved that the sequence of arithmetic means of independent and identically distributed (i.i.d.) random variables converges completely to the expected value if the variance of the summands is finite. Erdös [2] proved the converse. The result of HsuRobbins-Erdös is a fundamental theorem in probability theory and has been generalized and extended in several directions by many authors. One of the most important generalizations is Baum and Katz [3] for the strong law of large numbers.

Recently, Baek et al. [4] discussed the complete convergence of weighted sums for arrays of rowwise negatively associated random variables and obtained the following result. 
Theorem 1.1. Let $\left\{X_{n i}, i \geq 1, n \geq 1\right\}$ be an array of rowwise negatively associated random variables with $E X_{n i}=0$ and $P\left(\left|X_{n i}\right|>x\right) \leq C P(|X|>x)$ for all $i \geq 1, n \geq 1$ and $x \geq 0$. Suppose that $\beta \geq-1$, and that $\left\{a_{n i}, i \geq 1, n \geq 1\right\}$ be an array of constants such that

$$
\begin{aligned}
& \sup _{i \geq 1}\left|a_{n i}\right|=O\left(n^{-r}\right) \quad \text { for some } r>0, \\
& \sum_{i=1}^{\infty}\left|a_{n i}\right|=O\left(n^{\alpha}\right) \quad \text { for some } \alpha \in[0, r) .
\end{aligned}
$$

(i) If $1+\alpha+\beta>0$ and there exists some $\delta>0$ such that $\alpha / r+1<\delta \leq 2$ and $s=\max (1+((1+\alpha+\beta) / r), \delta)$, then under $E|X|^{s}<\infty$, we have

$$
\sum_{n=1}^{\infty} n^{\beta} P\left(\left|\sum_{i=1}^{\infty} a_{n i} X_{n i}\right|>\varepsilon\right)<\infty \quad \forall \varepsilon>0 .
$$

(ii) If $1+\alpha+\beta=0$, then under $E(|X| \log (1+|X|))<\infty$, (1.2) remains true.

The main purpose of this paper is to generalize and improve the above results for arrays of rowwise negatively associated random variables to the case of asymptotically almost negatively associated random variables. In addition, we will also consider the case $1+\alpha+\beta<0$, which complements the result of Baek et al. [4] and $\mathrm{Wu}$ [5].

Definition 1.2. A finite collection of random variables $X_{1}, X_{2}, \ldots, X_{n}$ is said to be negatively associated (NA, in short) if for every pair of disjoint subsets $A_{1}, A_{2}$ of $\{1,2, \ldots, n\}$,

$$
\operatorname{Cov}\left\{f\left(X_{i}: i \in A_{1}\right), g\left(X_{j}: j \in A_{2}\right)\right\} \leq 0,
$$

whenever $f$ and $g$ are coordinatewise nondecreasing such that this covariance exists. An infinite sequence $\left\{X_{n}, n \geq 1\right\}$ is NA if every finite subcollection is NA.

An array of random variables $\left\{X_{n i}, i \geq 1, n \geq 1\right\}$ is called rowwise NA random variables if for every $n \geq 1,\left\{X_{n i}, i \geq 1\right\}$ is a sequence of NA random variables.

The concept of negative association was introduced by Block et al. [6] and carefully studied by Joag-Dev and Proschan [7]. By inspecting the proof of maximal inequality for the NA random variables in Matula [8], one also can allow negative correlations provided they are small. Primarily motivated by this, Chandra and Ghosal $[9,10]$ introduced the following dependence.

Definition 1.3. A sequence $\left\{X_{n}, n \geq 1\right\}$ of random variables is called asymptotically almost negatively associated (AANA, in short) if there exists a nonnegative sequence $q(n) \rightarrow 0$ as $n \rightarrow \infty$ such that

$$
\operatorname{Cov}\left(f\left(X_{n}\right), g\left(X_{n+1}, X_{n+2}, \ldots, X_{n+k}\right)\right) \leq q(n)\left[\operatorname{Var}\left(f\left(X_{n}\right)\right) \operatorname{Var}\left(g\left(X_{n+1}, X_{n+2}, \ldots, X_{n+k}\right)\right)\right]^{1 / 2},
$$

for all $n, k \geq 1$ and for all coordinatewise nondecreasing continuous functions $f$ and $g$, whenever the variances exist. 
An array of random variables $\left\{X_{n i}, i \geq 1, n \geq 1\right\}$ is called rowwise AANA random variables if for every $n \geq 1,\left\{X_{n i}, i \geq 1\right\}$ is a sequence of AANA random variables.

The family of AANA sequence contains NA (in particular, independent) sequences (with $q(n)=0, n \geq 1$ ) and some more sequences of random variables which are not much deviated from being negatively associated. An example of an AANA sequence which is not NA was constructed by Chandra and Ghosal [9].

Since the concept of AANA sequence was introduced by Chandra and Ghosal [9], many applications have been found. See for example, Chandra and Ghosal [9] derived the Kolmogorov type inequality and the strong law of large numbers of MarcinkiewiczZygmund, Chandra and Ghosal [10] obtained the almost sure convergence of weighted averages, Ko et al. [11] studied the Hájek-Rényi type inequality, Wang et al. [12] established the law of the iterated logarithm for product sums, Yuan and An [13] established some Rosenthal type inequalities for maximum partial sums of AANA sequence, and Wang et al. [14] obtained some strong growth rate and the integrability of supremum for the partial sums of AANA random variables, and so forth. Our aim is to further study the complete convergence of weighted sums for arrays of rowwise AANA random variables.

Throughout this paper, let $\left\{X_{n i}, i \geq 1, n \geq 1\right\}$ be an array of rowwise AANA random variables with the mixing coefficients $\{q(i), i \geq 1\}$ in each row. For $p>1$, let $q \doteq p /(p-1)$ be the dual number of $p$. The symbol $C$ denotes a positive constant which is not necessarily the same one in each appearance and $\lceil x\rceil$ denotes the integer part of $x$. For a finite set $A$, the symbol $\sharp A$ denotes the number of elements in the set $A$. Let $I(A)$ be the indicator function of the set $A$. $a_{n}=O\left(b_{n}\right)$ stands for $a_{n} \leq C b_{n}$. Denote $\log x=\ln \max (x, e), X^{+}=\max (X, 0)$ and $X^{-}=\max (-X, 0)$.

The paper is organized as follows. Three important lemmas are provided in Section 2. The main results and their proofs are presented in Section 3. We will provide some sufficient conditions for complete convergence for arrays of rowwise AANA random variables which are stochastically dominated by a random variable $X$.

\section{Preliminaries}

Firstly, we will give the definition of stochastic domination.

Definition 2.1. A sequence $\left\{X_{n}, n \geq 1\right\}$ of random variables is said to be stochastically dominated by a random variable $X$ if there exists a positive constant $C$ such that

$$
P\left(\left|X_{n}\right|>x\right) \leq C P(|X|>x),
$$

for all $x \geq 0$ and $n \geq 1$.

An array $\left\{X_{n i}, i \geq 1, n \geq 1\right\}$ of rowwise random variables is said to be stochastically dominated by a random variable $X$ if there exists a positive constant $C$ such that

$$
P\left(\left|X_{n i}\right|>x\right) \leq C P(|X|>x),
$$

for all $x \geq 0, i \geq 1$ and $n \geq 1$.

The proofs of the main results of the paper are based on the following three lemmas. 
Lemma 2.2 (cf. Yuan and An [13, Lemma 2.1]). Let $\left\{X_{n}, n \geq 1\right\}$ be a sequence of AANA random variables with mixing coefficients $\{q(n), n \geq 1\}$, let $f_{1}, f_{2}, \ldots$ be all nondecreasing (or all nonincreasing) and continuous functions, then $\left\{f_{n}\left(X_{n}\right), n \geq 1\right\}$ is still a sequence of AANA random variables with mixing coefficients $\{q(n), n \geq 1\}$.

Lemma 2.3 (cf. Yuan and An [13, Theorem 2.1]). Let $p>1$ and $\left\{X_{n}, n \geq 1\right\}$ be a sequence of zero mean random variables with mixing coefficients $\{q(n), n \geq 1\}$.

If $\sum_{n=1}^{\infty} q^{2}(n)<\infty$, then there exists a positive constant $C_{p}$ depending only on $p$ such that for all $n \geq 1$ and $1<p \leq 2$,

$$
E\left(\max _{1 \leq j \leq n}\left|\sum_{i=1}^{j} X_{i}\right|^{p}\right) \leq C_{p} \sum_{i=1}^{n} E\left|X_{i}\right|^{p}
$$

If $\sum_{n=1}^{\infty} q^{q / p}(n)<\infty$ for some $p \in\left(3 \cdot 2^{k-1}, 4 \cdot 2^{k-1}\right]$, where integer number $k \geq 1$, then there exists a positive constant $D_{p}$ depending only on $p$ such that for all $n \geq 1$,

$$
E\left(\max _{1 \leq j \leq n}\left|\sum_{i=1}^{j} X_{i}\right|^{p}\right) \leq D_{p}\left\{\sum_{i=1}^{n} E\left|X_{i}\right|^{p}+\left(\sum_{i=1}^{n} E X_{i}^{2}\right)^{p / 2}\right\} .
$$

Lemma 2.4. Let $\left\{X_{n i}, i \geq 1, n \geq 1\right\}$ be an array of rowwise random variables which is stochastically dominated by a random variable X. For any $\alpha>0$ and $b>0$, the following two statements hold:

$$
\begin{gathered}
E\left|X_{n i}\right|^{\alpha} I\left(\left|X_{n i}\right| \leq b\right) \leq C_{1}\left[E|X|^{\alpha} I(|X| \leq b)+b^{\alpha} P(|X|>b)\right] \\
E\left|X_{n i}\right|^{\alpha} I\left(\left|X_{n i}\right|>b\right) \leq C_{2} E|X|^{\alpha} I(|X|>b)
\end{gathered}
$$

where $C_{1}$ and $C_{2}$ are positive constants.

\section{Main Results}

In this section, we will study the complete convergence for weighted sums of arrays of rowwise AANA random variables. As applications, the Baum and Katz type result for arrays of rowwise AANA random variables and the Marcinkiewicz-Zygmund type strong law of large numbers for sequences of AANA random variables are obtained. Let $\left\{X_{n i}, i \geq 1, n \geq 1\right\}$ be an array of rowwise AANA random variables with the mixing coefficients $\{q(i), i \geq 1\}$ in each row and $\left\{a_{n i}, i \geq 1, n \geq 1\right\}$ be an array of real numbers. Let $\left\{X_{i}, i \geq 1\right\}$ be a sequence of AANA random variables with the mixing coefficients $\{q(i), i \geq 1\}$. Our main results are as follows.

Theorem 3.1. Suppose that $\beta \geq-1$. Let $\left\{X_{n i}, i \geq 1, n \geq 1\right\}$ be an array of rowwise AANA random variables, which is stochastically dominated by a random variable $X$, and $\left\{a_{n i}, i \geq 1, n \geq 1\right\}$ be an array of constants such that

$$
\begin{gathered}
\sup _{i \geq 1}\left|a_{n i}\right|=O\left(n^{-r}\right) \quad \text { for some } r>0, \\
\sum_{i=1}^{\infty}\left|a_{n i}\right|^{\theta}=O\left(n^{\alpha}\right) \quad \text { for some } 0<\theta<2 \text { and some } \alpha \text { such that } \theta+\frac{\alpha}{r}<2 .
\end{gathered}
$$


(i) Assume that $\sum_{n=1}^{\infty} q^{2}(n)<\infty$ when $1<\theta<2$. If $1+\alpha+\beta<0$ and $E|X|^{\theta}<\infty$, then

$$
\sum_{n=1}^{\infty} n^{\beta} P\left(\max _{1 \leq j \leq n}\left|\sum_{i=1}^{j} a_{n i} X_{n i}\right|>\varepsilon\right)<\infty \quad \forall \varepsilon>0
$$

and (1.2) holds.

(ii) If $1+\alpha+\beta>0, \beta>-1$ and

$$
E|X|^{s}<\infty, \quad \text { where } s=\theta+\frac{1+\alpha+\beta}{r},
$$

and assume further that $E X_{n i}=0$ and $\sum_{n=1}^{\infty} q^{q / p}(n)<\infty$ for some $p \in\left(3 \cdot 2^{k-1}, 4 \cdot 2^{k-1}\right]$ and

$$
p>\max \left(2, \frac{2(1+\beta)}{r(2-\theta)-\alpha}, s\right)
$$

when $s \geq 1$, where integer number $k \geq 1$, then (1.2) and (3.3) hold.

(iii) If $1+\alpha+\beta=0$ and

$$
E|X|^{\theta} \log |X|<\infty
$$

and assume further that $E X_{n i}=0$ and $\sum_{n=1}^{\infty} q^{2}(n)<\infty$ when $1 \leq \theta<2$, then (1.2) and (3.3) hold.

Proof. The proof of (1.2) is similar to that of (3.3), so we only prove (3.3). Without loss of generality, we assume that $a_{n i}>0$ for all $i \geq 1$ and $n \geq 1$ (Otherwise, we use $a_{n i}^{+}$and $a_{n i}^{-}$instead of $a_{n i}$, resp., and note that $a_{n i}=a_{n i}^{+}-a_{n i}^{-}$). From the conditions (3.1) and (3.2), we assume that

$$
\sup _{i \geq 1} a_{n i}=n^{-r}, \quad \sum_{i=1}^{\infty} a_{n i}^{\theta}=n^{\alpha}, \quad n \geq 1 .
$$

(i) If $1+\alpha+\beta<0$, then the result can be easily proved by the following:

$$
\begin{aligned}
\sum_{n=1}^{\infty} n^{\beta} P\left(\max _{1 \leq j \leq n}\left|\sum_{i=1}^{j} a_{n i} X_{n i}\right|>\varepsilon\right) & \leq C \sum_{n=1}^{\infty} n^{\beta} E\left(\max _{1 \leq j \leq n}\left|\sum_{i=1}^{j} a_{n i} X_{n i}\right|^{\theta}\right) \\
& \leq C \sum_{n=1}^{\infty} n^{\beta} \sum_{i=1}^{n} E\left|a_{n i} X_{n i}\right|^{\theta} \\
& \leq C \sum_{n=1}^{\infty} n^{\alpha+\beta} E|X|^{\theta}<\infty .
\end{aligned}
$$

In the following, we will prove the result when $1+\alpha+\beta \geq 0$. Denote

$$
X_{n i}^{\prime}=-I\left(a_{n i} X_{n i}<-1\right)+a_{n i} X_{n i} I\left(\left|a_{n i} X_{n i}\right| \leq 1\right)+I\left(a_{n i} X_{n i}>1\right), \quad i \geq 1, n \geq 1 .
$$


Thus, $\left\{X_{n i}^{\prime}, i \geq 1, n \geq 1\right\}$ is still an array of rowwise AANA random variables with the mixing coefficients $\{q(i), i \geq 1\}$ in each row by Lemma 2.2. It is easy to check that for any $\varepsilon>0$,

$$
\left(\max _{1 \leq j \leq n}\left|\sum_{i=1}^{j} a_{n i} X_{n i}\right|>\varepsilon\right) \subset \bigcup_{i=1}^{n}\left(\left|a_{n i} X_{n i}\right|>1\right) \bigcup\left(\max _{1 \leq j \leq n}\left|\sum_{i=1}^{j} X_{n i}^{\prime}\right|>\varepsilon\right),
$$

which implies that

$$
\begin{aligned}
\sum_{n=1}^{\infty} n^{\beta} P\left(\max _{1 \leq j \leq n}\left|\sum_{i=1}^{j} a_{n i} X_{n i}\right|>\varepsilon\right) \leq & \sum_{n=1}^{\infty} n^{\beta} \sum_{i=1}^{n} P\left(\left|a_{n i} X_{n i}\right|>1\right) \\
& +\sum_{n=1}^{\infty} n^{\beta} P\left(\max _{1 \leq j \leq n}\left|\sum_{i=1}^{j} X_{n i}^{\prime}\right|>\varepsilon\right) \\
\doteq & I+J .
\end{aligned}
$$

Hence, in order to prove (3.3), it suffices to prove that $I<\infty$ and $J<\infty$.

(ii) If $1+\alpha+\beta>0$, then by Markov's inequality, (3.7) and $E|X|^{s}<\infty$, we can get that

$$
\begin{aligned}
\sum_{n=1}^{\infty} n^{\beta} \sum_{i=1}^{n} P\left(\left|a_{n i} X_{n i}\right|>1\right) & \leq C \sum_{n=1}^{\infty} n^{\beta} \sum_{i=1}^{n} P\left(\left|a_{n i} X\right|>1\right) \\
& \leq C \sum_{n=1}^{\infty} n^{\beta} \sum_{i=1}^{n} a_{n i}^{\theta} E|X|^{\theta} I\left(|X|>\frac{1}{a_{n i}}\right) \\
& \leq C \sum_{n=1}^{\infty} n^{\alpha+\beta} E|X|^{\theta} I\left(|X|>n^{r}\right) \\
& \leq C \sum_{n=1}^{\infty} n^{\alpha+\beta} \sum_{k=n}^{\infty} E|X|^{\theta} I\left(k^{r} \leq|X|<(k+1)^{r}\right) \\
& =C \sum_{k=1}^{\infty} \sum_{n=1}^{k} n^{\alpha+\beta} E|X|^{\theta} I\left(k^{r} \leq|X|<(k+1)^{r}\right) \\
& \leq C \sum_{k=1}^{\infty} k^{1+\alpha+\beta} E|X|^{\theta} I\left(k^{r} \leq|X|<(k+1)^{r}\right) \\
& \leq C \sum_{k=1}^{\infty} E|X|^{\theta+(1+\alpha+\beta) / r} I\left(k^{r} \leq|X|<(k+1)^{r}\right) \\
& \leq C E|X|^{\theta+(1+\alpha+\beta) / r}<\infty
\end{aligned}
$$

which implies that $I<\infty$.

Next, we will prove that $J<\infty$ for $s \geq 1$ and $s<1$, respectively. 
Case $1(s \geq 1)$. Firstly, we will show that

$$
\max _{1 \leq j \leq n}\left|\sum_{i=1}^{j} E X_{n i}^{\prime}\right| \longrightarrow 0, \quad \text { as } n \longrightarrow \infty .
$$

Actually, by the conditions $E X_{n i}=0$, Lemma 2.4, (3.7), and $E|X|^{s}<\infty$, we have that

$$
\begin{aligned}
\max _{1 \leq j \leq n}\left|\sum_{i=1}^{j} E X_{n i}^{\prime}\right| & \leq \max _{1 \leq j \leq n}\left|\sum_{i=1}^{j} E a_{n i} X_{n i} I\left(\left|a_{n i} X_{n i}\right| \leq 1\right)\right|+\sum_{i=1}^{n} P\left(\left|a_{n i} X_{n i}\right|>1\right) \\
& =\max _{1 \leq j \leq n}\left|\sum_{i=1}^{j} E a_{n i} X_{n i} I\left(\left|a_{n i} X_{n i}\right|>1\right)\right|+\sum_{i=1}^{n} P\left(\left|a_{n i} X_{n i}\right|>1\right) \\
& \leq C \sum_{i=1}^{n} E\left|a_{n i} X_{n i}\right|^{s} I\left(\left|a_{n i} X_{n i}\right|>1\right) \leq C \sum_{i=1}^{n} a_{n i}^{s} E|X|^{s} I\left(|X|>\frac{1}{a_{n i}}\right) \\
& \leq C\left(\sup _{i \geq 1} a_{n i}\right)^{s-\theta} \sum_{i=1}^{n} a_{n i}^{\theta} E|X|^{s} I\left(|X|>n^{r}\right) \\
& \leq C\left(n^{-r}\right)^{s-\theta} n^{\alpha} E|X|^{s} I\left(|X|>n^{r}\right) \\
& =C n^{-(1+\beta)} E|X|^{s} I\left(|X|>n^{r}\right) \longrightarrow 0, \quad \text { as } n \longrightarrow \infty,
\end{aligned}
$$

which implies (3.13). Hence, to prove $J<\infty$, we only need to show that for all $\varepsilon>0$,

$$
J^{*} \doteq \sum_{n=1}^{\infty} n^{\beta} P\left(\max _{1 \leq j \leq n}\left|\sum_{i=1}^{j}\left(X_{n i}^{\prime}-E X_{n i}^{\prime}\right)\right|>\frac{\varepsilon}{2}\right)<\infty .
$$

By Markov's inequality, Lemma 2.3, $C_{r}$ 's inequality, and Jensen's inequality, we have for $p \geq 2$ that

$$
\begin{aligned}
J^{*} & \leq C \sum_{n=1}^{\infty} n^{\beta} E\left(\max _{1 \leq j \leq n}\left|\sum_{i=1}^{j}\left(X_{n i}^{\prime}-E X_{n i}^{\prime}\right)\right|^{p}\right) \\
& \leq C \sum_{n=1}^{\infty} n^{\beta}\left[\left(\sum_{i=1}^{n} E\left|X_{n i}^{\prime}\right|^{2}\right)^{p / 2}+\sum_{i=1}^{n} E\left|X_{n i}^{\prime}\right|^{p}\right] \\
& \doteq J_{1}+J_{2} .
\end{aligned}
$$

Take

$$
p>\max \left(2, \frac{2(1+\beta)}{r(2-\theta)-\alpha}, \theta+\frac{1+\alpha+\beta}{r}\right),
$$


which implies that $\beta-[r(2-\theta)-\alpha] p / 2<-1$ and $\alpha+\beta-r(p-\theta)<-1$. By $C_{r}$ 's inequality and Lemma 2.4, we can get

$$
\begin{aligned}
J_{1} & \doteq C \sum_{n=1}^{\infty} n^{\beta}\left(\sum_{i=1}^{n} E\left|X_{n i}^{\prime}\right|^{2}\right)^{p / 2} \\
& \leq C \sum_{n=1}^{\infty} n^{\beta}\left[\sum_{i=1}^{n} P\left(\left|a_{n i} X\right|>1\right)+\sum_{i=1}^{n} E\left|a_{n i} X\right|^{2} I\left(\left|a_{n i} X\right| \leq 1\right)\right]^{p / 2} .
\end{aligned}
$$

If $1 \leq s<2$, then by Markov's inequality, $E|X|^{s}<\infty$, and (3.7), we have

$$
\begin{aligned}
J_{1} & \leq C \sum_{n=1}^{\infty} n^{\beta}\left(\sum_{i=1}^{n} a_{n i}^{s} E|X|^{S}\right)^{p / 2} \\
& \leq C \sum_{n=1}^{\infty} n^{\beta}\left[\left(\sup _{i \geq 1} a_{n i}\right)^{s-\theta} \sum_{i=1}^{n} a_{n i}^{\theta}\right]^{p / 2} \leq C \sum_{n=1}^{\infty} n^{\beta}\left[n^{-r(s-\theta)} \cdot n^{\alpha}\right]^{p / 2} \\
& =C \sum_{n=1}^{\infty} n^{\beta-(((1+\beta) p) / 2)}<\infty \quad(\text { since } 1+\beta>0) .
\end{aligned}
$$

If $s \geq 2$, then by Markov's inequality, $E|X|^{s}<\infty$, and (3.7) again, we have

$$
\begin{aligned}
J_{1} & \leq C \sum_{n=1}^{\infty} n^{\beta}\left(\sum_{i=1}^{n} a_{n i}^{2} E|X|^{2}\right)^{p / 2} \\
& \leq C \sum_{n=1}^{\infty} n^{\beta}\left[\left(\sup _{i \geq 1} a_{n i}\right)^{2-\theta} \sum_{i=1}^{n} a_{n i}^{\theta}\right]^{p / 2} \\
& \leq C \sum_{n=1}^{\infty} n^{\beta}\left[n^{-r(2-\theta)} \cdot n^{\alpha}\right]^{p / 2}=C \sum_{n=1}^{\infty} n^{\beta-[r(2-\theta)-\alpha] p / 2}<\infty .
\end{aligned}
$$

From (3.18)-(3.20), we have proved that $J_{1}<\infty$.

By Lemma 2.4 again and the definition of stochastic domination, we can see that

$$
\begin{aligned}
J_{2} & \doteq C \sum_{n=1}^{\infty} n^{\beta} \sum_{i=1}^{n} E\left|X_{n i}^{\prime}\right|^{p} \\
& \leq C \sum_{n=1}^{\infty} n^{\beta} \sum_{i=1}^{n}\left[E\left|a_{n i} X_{n i}\right|^{p} I\left(\left|a_{n i} X_{n i}\right| \leq 1\right)+P\left(\left|a_{n i} X_{n i}\right|>1\right)\right] \\
& \leq C \sum_{n=1}^{\infty} n^{\beta} \sum_{i=1}^{n} P\left(\left|a_{n i} X\right|>1\right)+C \sum_{n=1}^{\infty} n^{\beta} \sum_{i=1}^{n} E\left|a_{n i} X\right|^{p} I\left(\left|a_{n i} X\right| \leq 1\right) \\
& \doteq J_{3}+J_{4} .
\end{aligned}
$$


$J_{3}<\infty$ has been proved by (3.12). In the following, we will show that $J_{4}<\infty$. Denote

$$
I_{n j}=\left\{i:(n j)^{r} \leq 1 / a_{n i}<[n(j+1)]^{r}\right\}, \quad n \geq 1, j \geq 1 .
$$

It is easily seen that $I_{n k} \bigcap I_{n j}=\emptyset$ for $k \neq j$ and $\bigcup_{j=1}^{\infty} I_{n j}=\mathbb{N}$ for all $n \geq 1$. Hence,

$$
\begin{aligned}
J_{4} \doteq & C \sum_{n=1}^{\infty} n^{\beta} \sum_{i=1}^{n} E\left|a_{n i} X\right|^{p} I\left(\left|a_{n i} X\right| \leq 1\right) \leq C \sum_{n=1}^{\infty} n^{\beta} \sum_{j=1}^{\infty} \sum_{i \in I_{n j}} E\left|a_{n i} X\right|^{p} I\left(\left|a_{n i} X\right| \leq 1\right) \\
\leq & C \sum_{n=1}^{\infty} n^{\beta} \sum_{j=1}^{\infty}\left(\sharp I_{n j}\right)(n j)^{-r p} E|X|^{p} I\left(|X| \leq[n(j+1)]^{r}\right) \\
\leq & C \sum_{n=1}^{\infty} n^{\beta} \sum_{j=1}^{\infty}\left(\sharp I_{n j}\right)(n j)^{-r p} \sum_{k=0}^{n(j+1)} E|X|^{p} I\left(k \leq|X|^{1 / r}<k+1\right) \\
= & C \sum_{n=1}^{\infty} n^{\beta} \sum_{j=1}^{\infty}\left(\sharp I_{n j}\right)(n j)^{-r p} \sum_{k=0}^{2 n} E|X|^{p} I\left(k \leq|X|^{1 / r}<k+1\right) \\
& +C \sum_{n=1}^{\infty} n^{\beta} \sum_{j=1}^{\infty}\left(\sharp I_{n j}\right)(n j)^{-r p} \sum_{k=2 n+1}^{n(j+1)} E|X|^{p} I\left(k \leq|X|^{1 / r}<k+1\right) \\
\doteq & J_{5}+J_{6} .
\end{aligned}
$$

It is easily seen that for all $m \geq 1$, we have that

$$
\begin{aligned}
n^{\alpha} & =\sum_{i=1}^{\infty} a_{n i}^{\theta}=\sum_{j=1}^{\infty} \sum_{i \in I_{n j}} a_{n i}^{\theta} \geq \sum_{j=1}^{\infty}\left(\sharp I_{n j}\right)[n(j+1)]^{-r \theta} \\
& \geq \sum_{j=m}^{\infty}\left(\sharp I_{n j}\right)[n(j+1)]^{-r \theta} \geq \sum_{j=m}^{\infty}\left(\sharp I_{n j}\right)[n(j+1)]^{-r \theta}\left[\frac{n(m+1)}{n(j+1)}\right]^{r(p-\theta)} \\
& =\sum_{j=m}^{\infty}\left(\sharp I_{n j}\right)[n(j+1)]^{-r p}[n(m+1)]^{r(p-\theta)},
\end{aligned}
$$

which implies that for all $m \geq 1$,

$$
\sum_{j=m}^{\infty}\left(\sharp I_{n j}\right)(n j)^{-r p} \leq C n^{\alpha} \cdot n^{-r(p-\theta)} \cdot m^{-r(p-\theta)}=C n^{\alpha-r(p-\theta)} \cdot m^{-r(p-\theta)} .
$$

Therefore,

$$
\begin{aligned}
J_{5} & \doteq C \sum_{n=1}^{\infty} n^{\beta} \sum_{j=1}^{\infty}\left(\sharp I_{n j}\right)(n j)^{-r p} \sum_{k=0}^{2 n} E|X|^{p} I\left(k \leq|X|^{1 / r}<k+1\right) \\
& \leq C \sum_{n=1}^{\infty} n^{\beta} \cdot n^{\alpha-r(p-\theta)} \sum_{k=0}^{2 n} E|X|^{p} I\left(k \leq|X|^{1 / r}<k+1\right)
\end{aligned}
$$




$$
\begin{aligned}
& \leq C \sum_{k=0}^{2} \sum_{n=1}^{\infty} n^{\alpha+\beta-r(p-\theta)} E|X|^{p} I\left(k \leq|X|^{1 / r}<k+1\right) \\
& +C \sum_{k=2}^{\infty} \sum_{n=\lceil k / 2\rceil}^{\infty} n^{\alpha+\beta-r(p-\theta)} E|X|^{p} I\left(k \leq|X|^{1 / r}<k+1\right) \\
& \leq C+C \sum_{k=2}^{\infty} k^{1+\alpha+\beta-r(p-\theta)} E|X|^{p} I\left(k \leq|X|^{1 / r}<k+1\right) \\
& \leq C+C \sum_{k=2}^{\infty} E|X|^{p+((1+\alpha+\beta) / r)-(p-\theta)} I\left(k \leq|X|^{1 / r}<k+1\right) \\
& \leq C+C E|X|^{\theta+((1+\alpha+\beta) / r)}<\infty, \\
& J_{6} \doteq C \sum_{n=1}^{\infty} n^{\beta} \sum_{j=1}^{\infty}\left(\sharp I_{n j}\right)(n j)^{-r p} \sum_{k=2 n+1}^{n(j+1)} E|X|^{p} I\left(k \leq|X|^{1 / r}<k+1\right) \\
& \leq C \sum_{n=1}^{\infty} n^{\beta} \sum_{k=2 n+1}^{\infty} \sum_{j \geq(k / n)-1}\left(\sharp I_{n j}\right)(n j)^{-r p} E|X|^{p} I\left(k \leq|X|^{1 / r}<k+1\right) \\
& \leq C \sum_{n=1}^{\infty} n^{\beta} \sum_{k=2 n+1}^{\infty} n^{\alpha-r(p-\theta)}\left(\frac{k}{n}\right)^{-r(p-\theta)} E|X|^{p} I\left(k \leq|X|^{1 / r}<k+1\right) \\
& \leq C \sum_{k=2}^{\infty} \sum_{n=1}^{\lceil k / 2\rceil} n^{\alpha+\beta} \cdot k^{-r(p-\theta)} E|X|^{p} I\left(k \leq|X|^{1 / r}<k+1\right) \\
& \leq C \sum_{k=2}^{\infty} k^{1+\alpha+\beta-r(p-\theta)} E|X|^{p} I\left(k \leq|X|^{1 / r}<k+1\right) \\
& \leq C \sum_{k=2}^{\infty} E|X|^{p+((1+\alpha+\beta) / r)-(p-\theta)} I\left(k \leq|X|^{1 / r}<k+1\right) \\
& \leq C E|X|^{\theta+((1+\alpha+\beta) / r)}<\infty .
\end{aligned}
$$

Thus, the inequality (3.15) follows from (3.16)-(3.21), (3.23), (3.26), and (3.27). The desired result (3.3) follows from (3.11), (3.12), and (3.15), immediately.

Case $2(s<1)$. We take $p>0$ such that $\theta+(1+\alpha+\beta) / r=s<p<1$, which implies that $\alpha+\beta-r(p-\theta)<-1$. By Markov's inequality and $C_{r}$ 's inequality, we have

$$
\begin{aligned}
J & \doteq \sum_{n=1}^{\infty} n^{\beta} P\left(\max _{1 \leq j \leq n}\left|\sum_{i=1}^{j} X_{n i}^{\prime}\right|>\varepsilon\right) \leq C \sum_{n=1}^{\infty} n^{\beta} E\left(\max _{1 \leq j \leq n}\left|\sum_{i=1}^{j} X_{n i}^{\prime}\right|\right)^{p} \\
& \leq C \sum_{n=1}^{\infty} n^{\beta} \sum_{i=1}^{n} E\left|X_{n i}^{\prime}\right|^{p} .
\end{aligned}
$$

The rest proof is similar to the process of $J_{2}<\infty$ in Case 1 , so we omit the details. 
(iii) If $1+\alpha+\beta=0$, then by Markov's inequality, (3.6), and similar to the process of (3.12), we can get that

$$
\begin{aligned}
I & \doteq \sum_{n=1}^{\infty} n^{\beta} \sum_{i=1}^{n} P\left(\left|a_{n i} X_{n i}\right|>1\right) \\
& \leq C \sum_{k=1}^{\infty} \sum_{n=1}^{k} n^{-1} E|X|^{\theta} I\left(k^{r} \leq|X|<(k+1)^{r}\right) \\
& \leq C \sum_{k=1}^{\infty} \log k E|X|^{\theta} I\left(k^{r} \leq|X|<(k+1)^{r}\right) \\
& \leq C \sum_{k=1}^{\infty} E|X|^{\theta} \log |X| I\left(k^{r} \leq|X|<(k+1)^{r}\right) \\
& \leq C E|X|^{\theta} \log |X|<\infty .
\end{aligned}
$$

Hence, to prove (3.3), we only need to show $J<\infty$. We will still consider the Cases $s \geq 1$ and $s<1$. Here, $s=\theta$.

Case $1(s \geq 1)$. Since $1+\alpha+\beta=0,1+\beta=-\alpha \geq 0$ and $s=\theta$, it follows that (3.14) still holds. Thus, it suffices to show that $J^{*}<\infty$.

By Markov's inequality, $C_{r}$ 's inequality, Lemma 2.3, Lemma 2.4, and (3.29), we have

$$
\begin{aligned}
J^{*} & \doteq \sum_{n=1}^{\infty} n^{\beta} P\left(\max _{1 \leq j \leq n}\left|\sum_{i=1}^{j}\left(X_{n i}^{\prime}-E X_{n i}^{\prime}\right)\right|>\frac{\varepsilon}{2}\right) \\
& \leq C \sum_{n=1}^{\infty} n^{\beta} E\left(\max _{1 \leq j \leq n}\left|\sum_{i=1}^{j}\left(X_{n i}^{\prime}-E X_{n i}^{\prime}\right)\right|^{2}\right) \leq C \sum_{n=1}^{\infty} n^{\beta} \sum_{i=1}^{n} E\left|X_{n i}^{\prime}\right|^{2} \\
& \leq C \sum_{n=1}^{\infty} n^{\beta} \sum_{i=1}^{n} P\left(\left|a_{n i} X\right|>1\right)+C \sum_{n=1}^{\infty} n^{\beta} \sum_{i=1}^{n} E\left|a_{n i} X\right|^{2} I\left(\left|a_{n i} X\right| \leq 1\right) \\
& \leq C+J_{5}^{*}+J_{6}^{*} .
\end{aligned}
$$

Here, $J_{5}^{*}$ and $J_{6}^{*}$ are $J_{5}$ and $J_{6}$ when $p=2$ in (ii), respectively. Notice that $\alpha+\beta=-1$ and $\alpha+\beta-r(2-\theta)<-1$, similar to the proof of $J_{5}<\infty$, we have

$$
J_{5}^{*} \leq C+C E|X|^{\theta}<\infty,
$$

and similar to the proof of $J_{6}<\infty$, we have

$$
\begin{aligned}
J_{6}^{*} & \leq C \sum_{k=2}^{\infty} \sum_{n=1}^{[k / 2]} n^{-1} \cdot k^{-r(2-\theta)} E|X|^{2} I\left(k \leq|X|^{1 / r}<k+1\right) \\
& \leq C \sum_{k=2}^{\infty} \log k \cdot k^{-r(2-\theta)} E|X|^{2} I\left(k \leq|X|^{1 / r}<k+1\right) \\
& \leq C E|X|^{\theta} \log |X|<\infty .
\end{aligned}
$$

Thus, $J^{*}<\infty$ follows from (3.30)-(3.32), immediately. 
Case $2(s<1)$. The process of the proof is similar to that of Case 2 in (ii). We only need to show that $J_{5}<\infty$ and $J_{6}<\infty$. Actually, similar to the proof of (3.26), we have

$$
J_{5} \leq C+C E|X|^{\theta}<\infty,
$$

and similar to the proof of (3.27), we have

$$
J_{6} \leq C E|X|^{\theta} \log |X|<\infty .
$$

This completes the proof of the theorem.

Remark 3.2. It is easily seen that the conditions (3.2), (3.4), and (3.6) in Theorem 3.1 are more general than the corresponding ones in Theorem 1.1. So Theorem 3.1 generalizes and improves the corresponding results of Theorem 3.1 in Baek et al. [4]. In addition, we not only consider the cases $1+\alpha+\beta>0$ and $1+\alpha+\beta=0$, we also consider the case $1+\alpha+\beta<0$. This complements the corresponding result of Baek et al. [4] and Wu [5].

By Theorems 3.1, we can extend the results of Baum and Katz [3] for independent and identically distributed random variables to the case of arrays of rowwise AANA random variables as follows.

Corollary 3.3. Let $\left\{X_{n i}, i \geq 1, n \geq 1\right\}$ be an array of rowwise AANA random variables which is stochastically dominated by a random variable $X$ and $E X_{n i}=0$ for all $i \geq 1, n \geq 1$.

and

(i) Let $\gamma>1$ and $1 \leq t<2$. If $E|X|^{r t}<\infty$ and $\sum_{n=1}^{\infty} q^{q / p}(n)<\infty$ for some $p \in\left(3 \cdot 2^{k-1}, 4 \cdot 2^{k-1}\right.$ ]

$$
p>\max \left(2, \frac{2 t(\gamma-1)}{2-t}, \gamma t\right)
$$

where integer number $k \geq 1$, then for all $\varepsilon>0$,

$$
\sum_{n=1}^{\infty} n^{\gamma-2} P\left(\max _{1 \leq j \leq n}\left|\sum_{i=1}^{j} X_{n i}\right|>\varepsilon n^{1 / t}\right)<\infty .
$$

(ii) If $E|X| \log |X|<\infty$ and $\sum_{n=1}^{\infty} q^{2}(n)<\infty$, then for all $\varepsilon>0$,

$$
\sum_{n=1}^{\infty} \frac{1}{n} P\left(\max _{1 \leq j \leq n}\left|\sum_{i=1}^{j} X_{n i}\right|>\varepsilon n\right)<\infty .
$$

Proof. (i) Let $a_{n i}=0$ if $i>n$ and $a_{n i}=n^{-1 / t}$ if $i \leq n$. Hence, conditions (3.1) and (3.2) hold for $\theta=1, r=1 / t$ and $\alpha=1-1 / t<r . \beta \doteq p-2>-1$. It is easy to check that

$$
1+\alpha+\beta=p-\frac{1}{t}>0, \quad 1+\frac{1+\alpha+\beta}{r}=p t \doteq s
$$

Therefore, the desired result (3.36) follows from Theorem 3.1(ii), immediately. 
(ii) Let $a_{n i}=0$ if $i>n$ and $a_{n i}=n^{-1}$ if $i \leq n$. Hence, conditions (3.1) and (3.2) hold for $r=-1, \theta=1$ and $\alpha=0$. Therefore, the desired result (3.37) follows from Theorem 3.1(iii), immediately. This completes the proof of the corollary.

Similar to the proofs of Theorem 3.1 and Corollary 3.3, we can get the Baum and Katz type result for sequences of AANA random variables as follows.

Theorem 3.4. Let $\left\{X_{n}, n \geq 1\right\}$ be a sequence of AANA random variables which is stochastically dominated by a random variable $X$ and $E X_{n}=0$ for $n \geq 1$.

(i) Let $\gamma>1$ and $1 \leq t<2$. If $E|X|^{r t}<\infty$ and $\sum_{n=1}^{\infty} q^{q / p}(n)<\infty$ for some $p \in\left(3 \cdot 2^{k-1}, 4 \cdot 2^{k-1}\right.$ ]

and

$$
p>\max \left(2, \frac{2 t(\gamma-1)}{2-t}, \gamma t\right),
$$

where integer number $k \geq 1$, then for all $\varepsilon>0$,

$$
\sum_{n=1}^{\infty} n^{\gamma-2} P\left(\max _{1 \leq j \leq n}\left|\sum_{i=1}^{j} X_{i}\right|>\varepsilon n^{1 / t}\right)<\infty .
$$

(ii) If $E|X| \log |X|<\infty$ and $\sum_{n=1}^{\infty} q^{2}(n)<\infty$, then for all $\varepsilon>0$,

$$
\sum_{n=1}^{\infty} \frac{1}{n} P\left(\max _{1 \leq j \leq n}\left|\sum_{i=1}^{j} X_{i}\right|>\varepsilon n\right)<\infty
$$

By Theorem 3.4, we can get the Marcinkiewicz-Zygmund type strong law of large numbers for AANA random variables as follows.

Corollary 3.5. Let $\left\{X_{n}, n \geq 1\right\}$ be a sequence of AANA random variables which is stochastically dominated by a random variable $X$ and $E X_{n}=0$ for $n \geq 1$.

(i) Let $\gamma>1$ and $1 \leq t<2$. If $E|X|^{r t}<\infty$ and $\sum_{n=1}^{\infty} q^{q / p}(n)<\infty$ for some $p \in\left(3 \cdot 2^{k-1}, 4 \cdot 2^{k-1}\right.$ ] and

$$
p>\max \left(2, \frac{2 t(\gamma-1)}{2-t}, \gamma t\right),
$$

where integer number $k \geq 1$, then

$$
n^{-1 / t} \sum_{i=1}^{n} X_{i} \longrightarrow 0 \quad \text { a.s. } n \longrightarrow \infty
$$

(ii) If $E|X| \log |X|<\infty$ and $\sum_{n=1}^{\infty} q^{2}(n)<\infty$, then

$$
\frac{1}{n} \sum_{i=1}^{n} X_{i} \longrightarrow 0 \quad \text { a.s. } n \longrightarrow \infty \text {. }
$$


Proof. (i) By (3.40), we can get that for all $\varepsilon>0$,

$$
\begin{aligned}
\infty & >\sum_{n=1}^{\infty} n^{\gamma-2} P\left(\max _{1 \leq j \leq n}\left|\sum_{i=1}^{j} X_{i}\right|>\varepsilon n^{1 / t}\right) \\
& =\sum_{k=0}^{\infty} \sum_{n=2^{k}}^{2^{k+1}-1} n^{\gamma-2} P\left(\max _{1 \leq j \leq n}\left|\sum_{i=1}^{j} X_{i}\right|>\varepsilon n^{1 / t}\right) \\
& \geq\left\{\begin{array}{l}
\sum_{k=0}^{\infty}\left(2^{k}\right)^{\gamma-2} 2^{k} P\left(\max _{1 \leq j \leq 2^{k}}\left|\sum_{i=1}^{j} X_{i}\right|>\varepsilon 2^{(k+1) / t}\right), \quad \text { if } \gamma \geq 2, \\
\sum_{k=0}^{\infty}\left(2^{k+1}\right)^{\gamma-2} 2^{k} P\left(\max _{1 \leq j \leq 2^{k}}\left|\sum_{i=1}^{j} X_{i}\right|>\varepsilon 2^{(k+1) / t}\right), \quad \text { if } 1<\gamma<2,
\end{array}\right. \\
& \geq\left\{\begin{array}{l}
\sum_{k=0}^{\infty} P\left(\max _{1 \leq j \leq 2^{k}}\left|\sum_{i=1}^{j} X_{i}\right|>\varepsilon 2^{(k+1) / t}\right), \quad \text { if } \gamma \geq 2, \\
\frac{1}{2} \sum_{k=0}^{\infty} P\left(\max _{1 \leq j \leq 2^{k}}\left|\sum_{i=1}^{j} X_{i}\right|>\varepsilon 2^{(k+1) / t}\right), \quad \text { if } 1<\gamma<2 .
\end{array}\right.
\end{aligned}
$$

By Borel-Cantelli Lemma, we obtain that

$$
\frac{\max _{1 \leq j \leq 2^{k}}\left|\sum_{i=1}^{j} X_{i}\right|}{2^{(k+1) / t}} \longrightarrow 0 \quad \text { a.s. } k \longrightarrow \infty
$$

For all positive integers $n$, there exists a positive integer $k_{0}$ such that $2^{k_{0}-1} \leq n<2^{k_{0}}$. We have by (3.46) that

$$
\frac{\left|\sum_{i=1}^{n} X_{i}\right|}{n^{1 / t}} \leq \max _{2^{k_{0}-1} \leq n<2^{k_{0}}} \frac{\left|\sum_{i=1}^{n} X_{i}\right|}{n^{1 / t}} \leq \frac{2^{2 / t} \max _{1 \leq j \leq 2^{k_{0}}}\left|\sum_{i=1}^{j} X_{i}\right|}{2^{k_{0}+1 / t}} \longrightarrow 0 \text { a.s. } k_{0} \longrightarrow \infty,
$$

which implies (3.43).

(ii) Similar to the proof of (i), we can get (ii), immediately. The details are omitted. This completes the proof of the corollary.

\section{Acknowledgments}

The authors are most grateful to the editor Irena Rachunková and anonymous referees for careful reading of the manuscript and valuable suggestions which helped in significantly improving an earlier version of this paper. This work was supported by the National Natural Science Foundation of China (11171001 and 11126176), Natural Science Foundation of Anhui Province (1208085QA03), Provincial Natural Science Research Project of Anhui Colleges (KJ2010A005), the Academic Innovation Team of Anhui University (KJTD001B), Doctoral Research Start-up Funds Projects of Anhui University, and the Talents Youth Fund of Anhui Province Universities (2011SQRL012ZD). 


\section{References}

[1] P. L. Hsu and H. Robbins, "Complete convergence and the law of large numbers," Proceedings of the National Academy of Sciences of the United States of America, vol. 33, no. 2, pp. 25-31, 1947.

[2] P. Erdös, "On a theorem of Hsu and Robbins," Annals of Mathematical Statistics, vol. 20, pp. 286-291, 1949.

[3] L. E. Baum and M. Katz, "Convergence rates in the law of large numbers," Transactions of the American Mathematical Society, vol. 120, no. 1, pp. 108-123, 1965.

[4] J.-I. Baek, I.-B. Choi, and S.-L. Niu, "On the complete convergence of weighted sums for arrays of negatively associated variables," Journal of the Korean Statistical Society, vol. 37, no. 1, pp. 73-80, 2008.

[5] Q. Y. Wu, "A complete convergence theorem for weighted sums of arrays of rowwise negatively dependent random variables," Journal of Inequalities and Applications, vol. 2012, no. 50, 2012.

[6] H. W. Block, T. H. Savits, and M. Shaked, "Some concepts of negative dependence," The Annals of Probability, vol. 10, no. 3, pp. 765-772, 1982.

[7] K. Joag-Dev and F. Proschan, "Negative association of random variables, with applications," Annals of Statistics, vol. 11, no. 1, pp. 286-295, 1983.

[8] P. Matuła, "A note on the almost sure convergence of sums of negatively dependent random variables," Statistics \& Probability Letters, vol. 15, no. 3, pp. 209-213, 1992.

[9] T. K. Chandra and S. Ghosal, "Extensions of the strong law of large numbers of Marcinkiewicz and Zygmund for dependent variables," Acta Mathematica Hungarica, vol. 71, no. 4, pp. 327-336, 1996.

[10] T. K. Chandra and S. Ghosal, "The strong law of large numbers for weighted averages under dependence assumptions," Journal of Theoretical Probability, vol. 9, no. 3, pp. 797-809, 1996.

[11] M.-H. Ko, T.-S. Kim, and Z. Lin, "The Hájeck-Rènyi inequality for the AANA random variables and its applications," Taiwan Journal of Mathematics, vol. 9, no. 1, pp. 111-122, 2005.

[12] Y. Wang, J. Yan, F. Cheng, and C. Su, "The strong law of large numbers and the law of the iterated logarithm for product sums of NA and AANA random variables," Southeast Asian Bulletin of Mathematics, vol. 27, no. 2, pp. 369-384, 2003.

[13] D. Yuan and J. An, "Rosenthal type inequalities for asymptotically almost negatively associated random variables and applications," Science in China A, vol. 52, no. 9, pp. 1887-1904, 2009.

[14] X. Wang, S. Hu, and W. Yang, "Convergence properties for asymptotically almost negatively associated sequence," Discrete Dynamics in Nature and Society, Article ID 218380, 15 pages, 2010. 


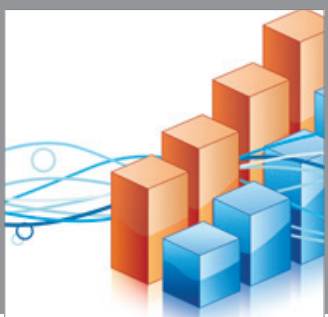

Advances in

Operations Research

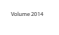

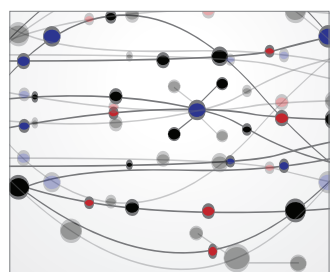

\section{The Scientific} World Journal
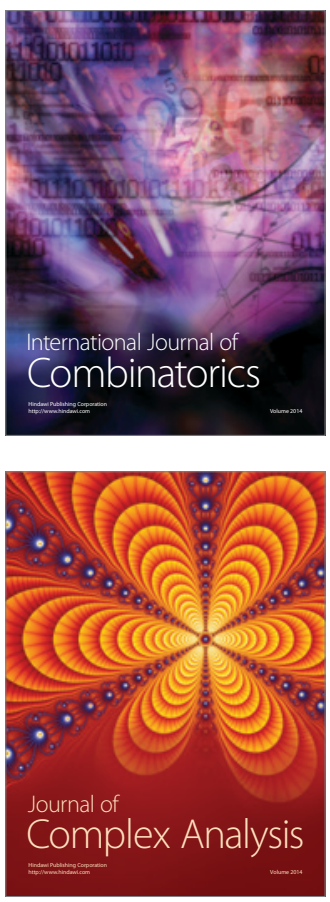

International Journal of

Mathematics and

Mathematical

Sciences
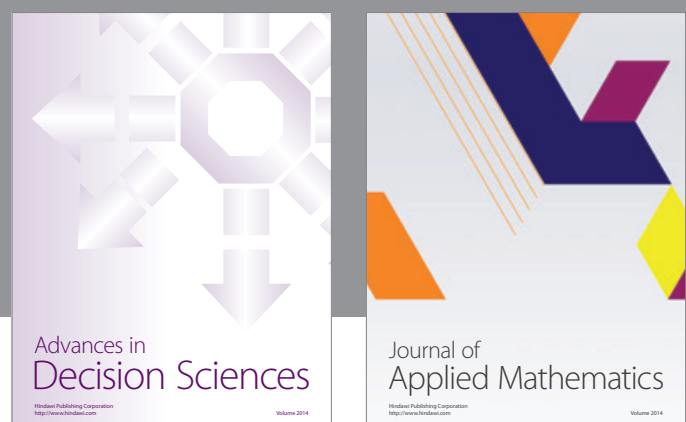

Journal of

Applied Mathematics
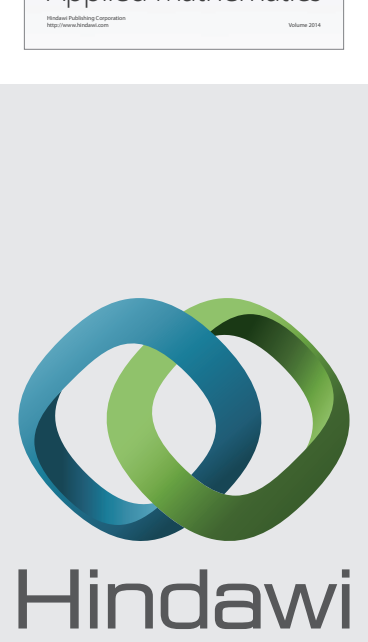

Submit your manuscripts at http://www.hindawi.com
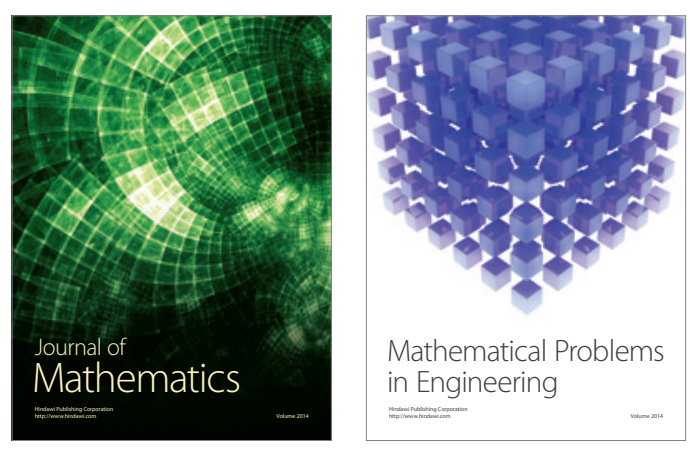

Mathematical Problems in Engineering
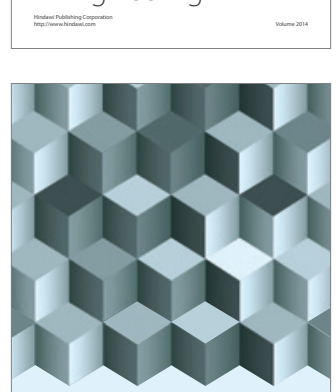

Journal of

Function Spaces
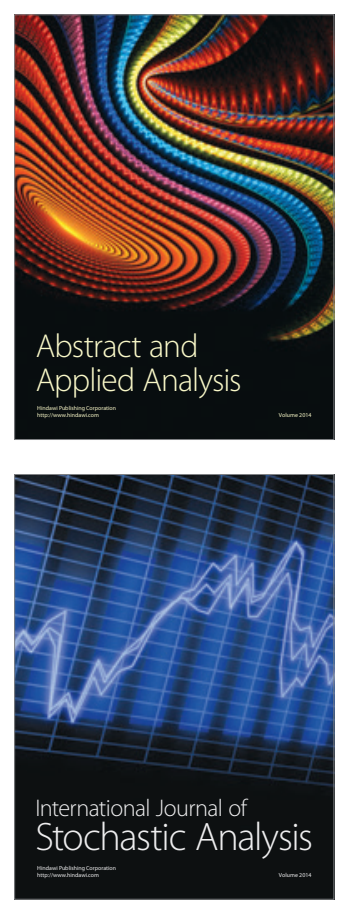

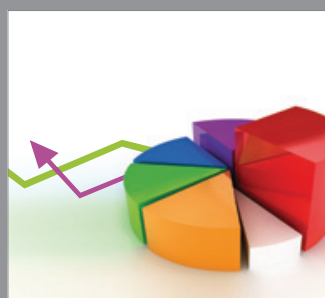

ournal of

Probability and Statistics

Promensencen
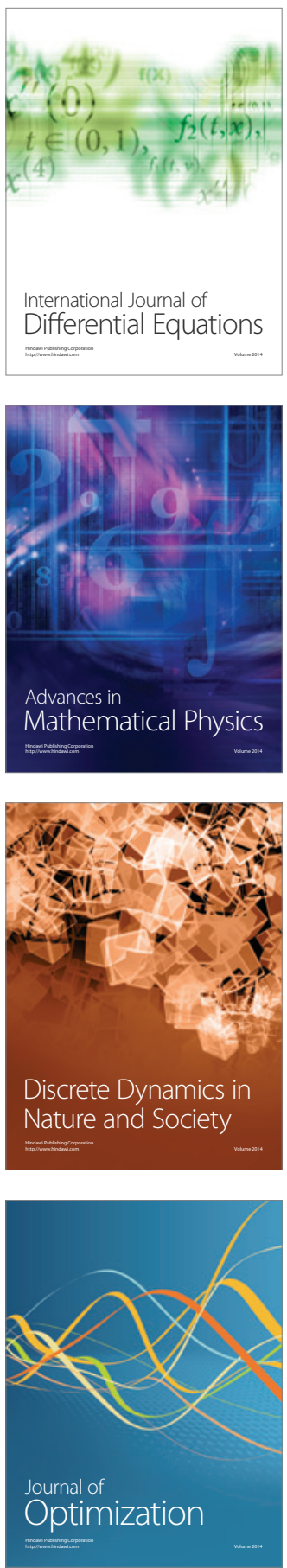